\title{
Primary Renal Synovial Sarcoma: Computed Tomography and Magnetic Resonance Imaging Findings
}

\author{
Xin-Ping Kuai ${ }^{1}$, Sheng-Yu Wang ${ }^{2}$, Chuan-Hai Jia ${ }^{1,{ }^{*}}$ and Jia-Ming Qiu ${ }^{3}$ \\ ${ }^{1}$ Department of Radiology, Changshu Second People's Hospital, Changshu, China \\ ${ }^{2}$ Department of Radiology, Ruijin Hospital North, School of Medicine, Shanghai Jiao Tong University, Shanghai, China \\ ${ }^{3}$ Department of Pathology, Changshu Second People's Hospital, Changshu, China \\ "Corresponding author: Department of Radiology, Changshu Second People’s Hospital, Changshu, China. Email: csjiachuanhai@163.com \\ Received 2018 July 16; Revised 2018 November 14; Accepted 2018 November 20.
}

\begin{abstract}
Synovial sarcomas (SS) are soft tissue neoplasms that usually occur in the vicinity of large joints. Herein, we present the case of a 48-year-old man with renal synovial sarcoma (RSS). The clinical manifestations, computed tomography (CT) scan and magnetic resonance imaging (MRI) features, microscopic characteristics, and immunohistochemical findings of this uncommon tumor are described.
\end{abstract}

Keywords: Synovial Sarcoma, Kidney, Computed Tomography, Magnetic Resonance Imaging

\section{Introduction}

Synovial sarcoma, a rare entity, approximately accounts for $5 \%-10 \%$ of grown-up soft tissue sarcomas and occurs primarily in periarticular regions of the extremities in youngsters (1). Primary renal synovial sarcoma(RSS) is extremely infrequent and some cases have always been contingently recorded since 1999 (2). In this study, we report the case of a 48-year-old man with RSS. The clinical manifestations, computed tomography(CT) scan and magnetic resonance imaging (MRI) features, microscopic characteristics, and immunohistochemical findings of this uncommon tumor are described.

\section{Case Presentation}

A 48-year-old man presented with interrupted lumbar pain for six months and macroscopic hematuria for 15 days. He was transferred to our hospital for evaluation of a renal mass, which was revealed by ultrasonography (US). Hypertension was diagnosed 10 years ago, which was controlled by Aprovel. Abdominal CT scan and MRI confirmed a heterogeneous soft tissue density or signal intensity mass located in the lower region of the right kidney, measuring approximately $8.8 \times 7.0 \times 8.0 \mathrm{~cm}$ (Figures 1 and 2 ). There was no evidence of retroperitoneal lymph node enlargement and tumor metastasis.
On unenhanced CT scan, the lesion appeared to have heterogeneous density with hemorrhage and without calcification. The density of the solid component of tumor was similar to that of the surrounding normal renal tissue. On contrast-enhanced CT scan, the mass presented as a solid-cystic lesion with the cyst located on the tumor margin. The tumor showed slightly heterogeneous enhancement. The CT values of the solid component of tumor were approximately 26 hounsfield unit (HU), $33 \mathrm{HU}, 35 \mathrm{HU}$, and $40 \mathrm{HU}$ on unenhanced, corticomedullary, nephrographic, and excretory phase images, respectively. On T1 and T2weighted imaging, the mass was shown to be heterogeneous, with the solid component of tumor having similar intensity to the surrounding normal renal tissue. On T1-weighted in phase and out-of-phase imaging, there was no change of signal intensity. Multiple cysts were clearly observed on T2-weighted and contrast-enhanced imaging. The tumor showed progressive enhancement and its apparent diffusion coefficient (ADC) value was $0.86 \times 10^{-3}$ $\mathrm{mm}^{2} / \mathrm{s}$.

Right radical nephrectomy was performed on the patient due to presumed diagnosis as right renal cell carcinoma.

On gross examination, the resected specimen measured $12 \times 8 \times 8 \mathrm{~cm}$. The tumor was excised from the lower pole of the right renal tissue. The cut surface of lesion 

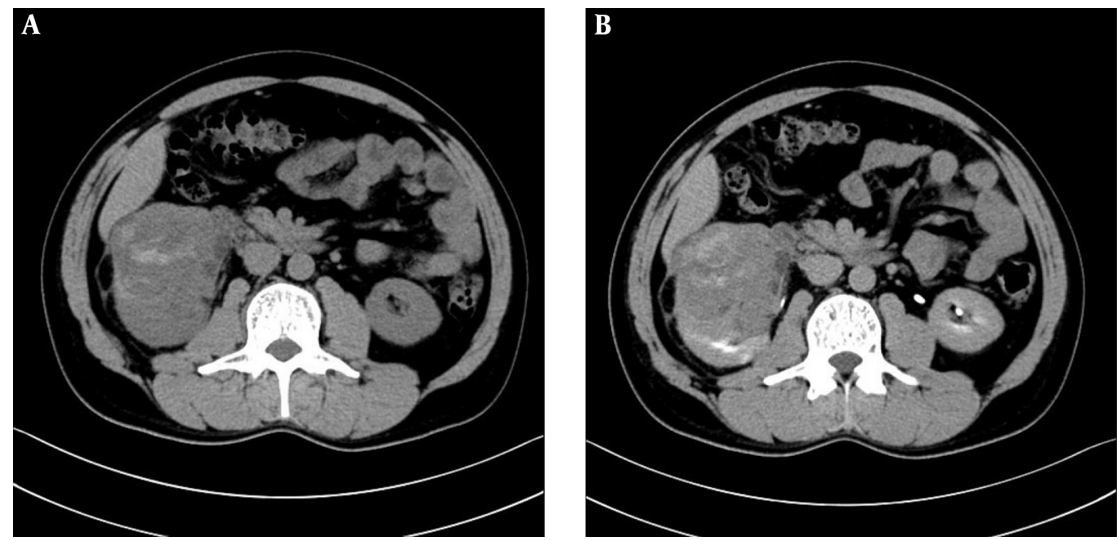

Figure 1. A 48-year-old man with interrupted lumbar pain. Abdominal computed tomography(CT) scan shows an $8.8 \times 7.0 \times 8.0 \mathrm{~cm}$ sized round, soft tissue density mass in the lower pole of the right kidney. Unenhanced (A) and excretory phase (B) CT scan shows a large mass, which distorted the renal architectures and heterogeneous enhancement of the solid components with unenhanced areas of hemorrhage and necrosis.
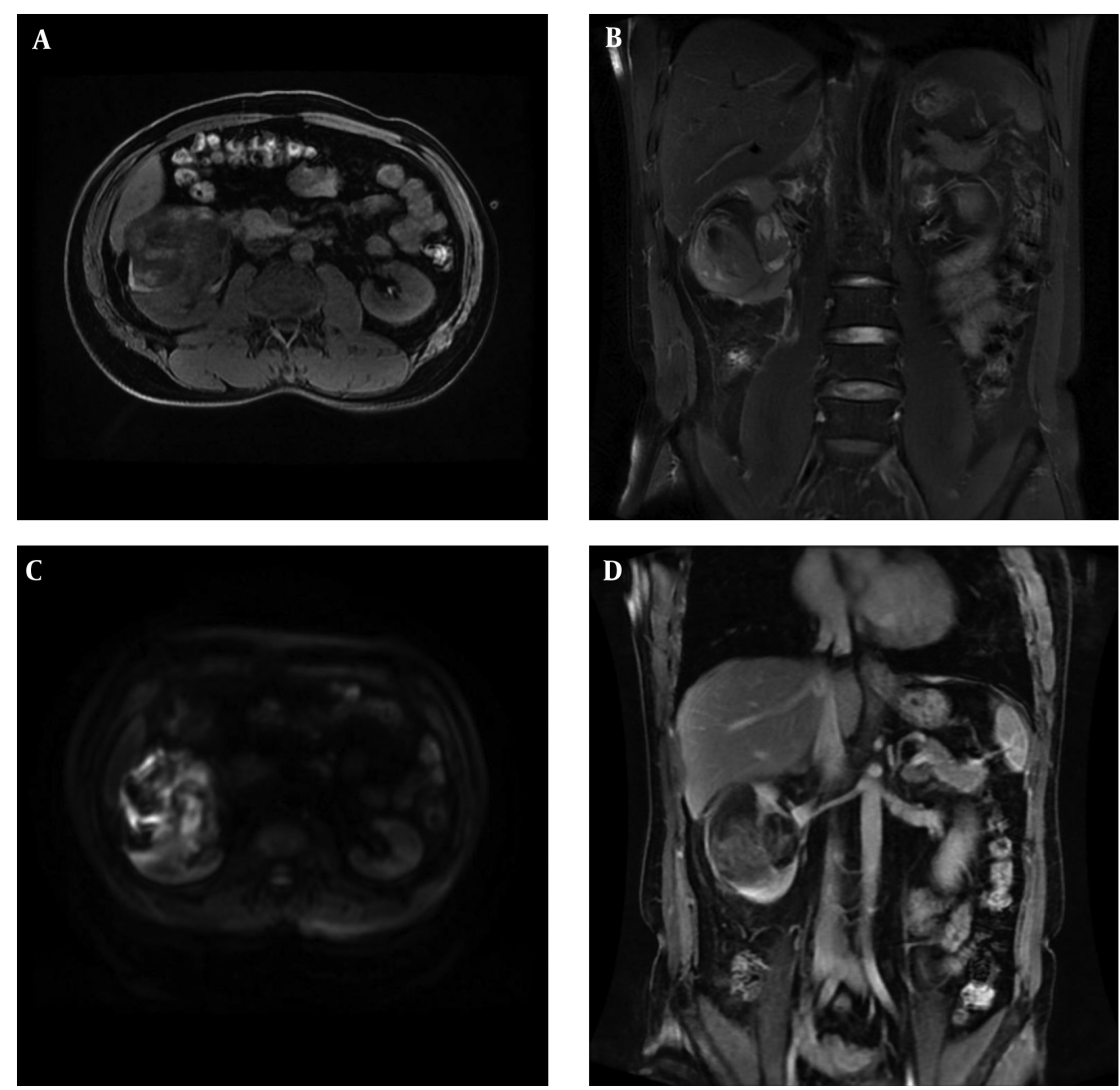

Figure 2. Magnetic resonance imaging (MRI) of the patient. On cross section T1-weighted image (A), the tumor is mixed hypointense, isointense and hyperintense. On corona T2-weighted image (B), mixed hperintense signal tumor with violation of perirenal fat is observed. On cross section diffusion-weighted (DW) image (C), marked hyperintense signal with focal hypointense is shown. Contrast-enhanced MRI (D) shows enhancement of solid component.

was of greyish-red and greyish-white soft solid component, accompanied by widespread regions of hemorrhage and necrosis. Histopathology revealed a neoplasm consisted of monomorphic spindle-shaped cells with scarce cyto- 

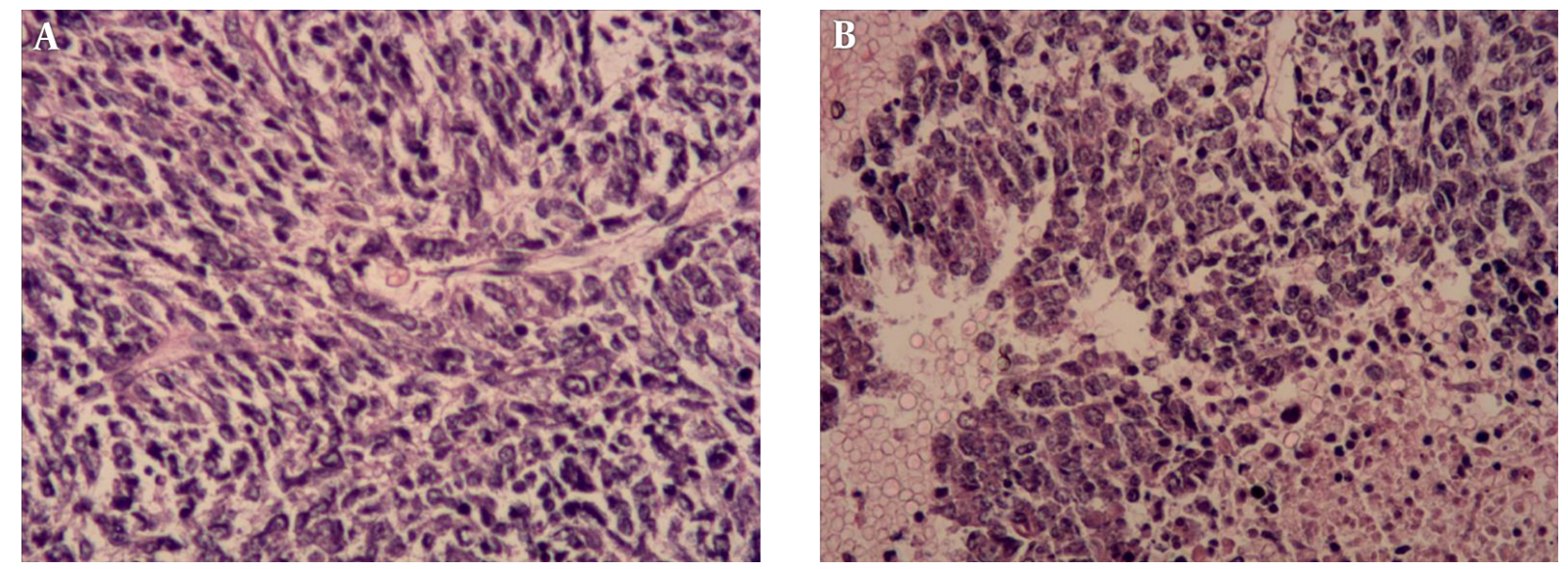

Figure 3. Histopathologic assessment of tumor. The tumor cells with unclear boundary and oval or irregular nuclei are spindle-shaped and arranged in fascicles (A, Hematoxylin-eosin $(\mathrm{H} \& \mathrm{E})$ staining $\times 200)$. Monomorphic, hyperchromatic, mitotically active spindle cells with hemorrhage and necrosis $(\mathrm{B}$, H\&E $\times 200)$.

plasms and ill-defined cell margins (Figure 3). Immunohistochemical staining showed that CD34, Desmin, Vimentin, epithelial membrane antigen (EMA) and smooth muscle actin (SMA) stains yielded negative results. Diffuse expression of $B C L-2$ and $C D 99$ and focal expression of $A E 1 / A E 3$ and EMA were detected in the tumor cells. Fluorescence in situ hybridization (FISH) assessment revealed no proof of translocation involvement (22q13) (SYT). Histological, immunohistochemical, and FISH features were compatible with monophasic synovial sarcoma with no SYT fusion.

The patient's postoperative recovery was uneventful and was discharged eight days after surgery with instructions of outpatient follow-up. The patient was not scheduled for any adjuvant chemotherapy. Four months after surgery, the patient was transferred to our hospital for fever, anuria, and tumor metastasis. After admission, the patient suffered from shock and electrolyte disorder and was given symptomatic treatments. The family members were informed that the prognosis was poor, and they asked for automatic discharge.

\section{Discussion}

Primary renal sarcoma is a rare tumor that accounts for approximately $1 \%$ - $3 \%$ of malignant renal neoplasms, and leiomyosarcoma is the most common subtype, which accounts for $50 \%-60 \%$ of all renal sarcomas (3). Primary RSS, a mesenchymal tumor occurring mainly in adults, is an extremely rare neoplasm $(3,4)$. It was described firstly by Argani et al. in 1999 and since then, approximately 120 cases of RSS have been reported in the literature $(2,5,6)$.
RSS can be initially misdiagnosed as renal cell carcinoma because of similar clinical characteristics (7). In previous reports, age of RSS patients ranged from 17 to 78 years (median: 36.5 years), and no gender predominance was observed $(8,9)$. Patients with RSS might be asymptomatic or suffer from abdominal pain, hematuria, fever, and dysuria (10). All sorts of presenting clinical characteristics may arise based on location and size of the lesion.

$\mathrm{CT}$ and MRI imaging provided useful information for determining the extent and invasiveness of the lesion. Because renal cell carcinoma (RCC) is the most common neoplasm, which accounts for approximately $80 \%$ of renal neoplasms (10), it is necessary to compare radiological characteristics of other tumors with that of RCC, for example clear cell RCC, the most common subtype. Although it is highly difficult to differentiate RSS from RCC based on clinical symptoms, some radiographic characteristics may be helpful for differential diagnosis.

In radiological literature, RSS has been described as large well-defined soft tissue masses with heterogeneous contrast enhancement (3). Some neoplasms may be dominantly cystic accompanied by enhancing septa and mural nodules (11). The solid component of the neoplasms revealed the 'rapid wash-in and slow wash-out' pattern of contrast enhancement on multiphase images, which is a distinctive feature in contrast to most common renal neoplasms (11). The heterogeneous signal intensity on T2weighted images is described as 'triple sign', which is represented by regions of low, intermediate, and high signal intensity $(3,12)$. However, clear cell RCC has peak enhancement on corticomedullary phase image and its necroses or cystic areas are located in the center of the tumor $(11,13)$. 
ADC value of clear cell RCC $\left(1.81 \times 10^{-3} \mathrm{~mm}^{2} / \mathrm{s}\right)$ is higher than that of non-clear cell RCC (14).

In our case, CT and MRI demonstrated a heterogeneous soft tissue density or intensity mass, and after intravenous contrast injection, we found slightly heterogeneous and progressive enhancement with hemorrhage and cysts. ADC value of this case was lower than that of clear cell RCC. The contiguous anatomical structures were infiltrated suggesting a malignant lesion. Similar to previously reported cases, the present case also presented a solid-cystic lesion with the cyst located on the tumor margin and'triple sign' on T2-weighted images $(3,11,12)$. Unlike previous cases, our case showed slight progressive enhancement $(3,11,12)$.

Macroscopically, RSS is a large solid mass with areas of hemorrhage, necrosis, and cyst. The histological finding in these cases demonstrate tumors composed of round spindle cells. Neoplastic cells are monomorphic, hyperchromatic, and mitotical (8). Synovial sarcomas can be divided into two subtypes, namely monophasic and biphasic, based on the absence or existence of well-developed glandular epithelium cells. In addition, the monophasic subtype is more frequent, but its prognosis is poorer (15).

Immunohistochemical analysis showed diffuse expression of BCL-2, which occurs in more than $98 \%$ of RSS cases. In addition, diffuse or focal immunoreactivity for CD99 is noted in about $60 \%$ of cases (15). Some synovial sarcomas express EMA as the only marker of epithelial differentiation. Synovial sarcomas are characterized by a specific, commonly reciprocal t (X;18) (p11.2; q11.2) translocation. Namely, the SS18 (formerly named SYT) gene (at 18q11) fuses with SSX genes $(8,15)$. In our case, FISH analysis showed no evidence of translocation involving SYT gene.

No definite medical therapy has been established for RSS due to its rarity. Radical nephrectomy was the first approach for patients to resolve symptoms and achieve local control of the disease (9). The prognosis for synovial sarcomas remains poor. In principle, chemotherapy is recommended, which mostly includes use of anthracyclines (adriamycin or epirubicin) and ifosfamide $(9,15)$.

\section{Footnotes}

Authors' Contributions: Xin-Ping Kuai participated in drafting the manuscript. Sheng-Yu Wang and Jia-Ming Qiu helped to collect the clinical data. Chuan-Hai Jia supervised the research coordination and design. All the authors approved the final manuscript.

Conflict of Interests: None declared.
Ethical Considerations: This case report was approved by our Institutional Review Board. Informed consent was acquired from individual participant.

Financial Disclosure: None declared.

Funding/Support: This study was funded by Changshu science and technology bureau (Grant No.cs201618) and Changshu health bureau (Grant No.csws201604).

\section{References}

1. Herzog CE. Overview of sarcomas in the adolescent and young adult population. J Pediatr Hematol Oncol. 2005;27(4):215-8. doi: 10.1097/01.mph.0000161762.53175.e4. [PubMed: 15838394].

2. Argani P, Faria PA, Epstein JI, Reuter VE, Perlman EJ, Beckwith JB, et al. Primary renal synovial sarcoma: Molecular and morphologic delineation of an entity previously included among embryonal sarcomas of the kidney. Am J Surg Pathol. 2000;24(8):1087-96. doi: 10.1097/00000478-200008000-00006. [PubMed:10935649].

3. Katabathina VS, Vikram R, Nagar AM, Tamboli P, Menias CO, Prasad SR. Mesenchymal neoplasms of the kidney in adults: Imaging spectrum with radiologic-pathologic correlation. Radiographics. 2010;30(6):1525-40. doi: 10.1148/rg.306105517. [PubMed: 21071373].

4. Moch H, Cubilla AL, Humphrey PA, Reuter VE, Ulbright TM. The 2016 WHO Classification of Tumours of the Urinary System and Male Genital Organs-Part A: Renal, Penile, and Testicular Tumours. Eur Urol. 2016;70(1):93-105. doi: 10.1016/j.eururo.2016.02.029. [PubMed: 26935559].

5. Dutt UK, Manikandan R, Dorairajan LN, Srinivas BH. Biphasic renal synovial sarcoma with extensive venous tumor thrombosis: A rare presentation. Urol Ann. 2018;10(3):339-41. doi: 10.4103/UA.UA_9_18. [PubMed: 30089998]. [PubMed Central: PMC6060589].

6. El Chediak A, Mukherji D, Temraz S, Nassif S, Sinno S, Mahfouz R, et al. Primary synovial sarcoma of the kidney: A case report of complete pathological response at a Lebanese tertiary care center. BMC Urol. 2018;18(1):40. doi: 10.1186/s12894-018-0358-z. [PubMed: 29751751]. [PubMed Central: PMC5948733].

7. Lopes H, Pereira CA, Zucca LE, Serrano SV, Silva SR, Camparoto ML, et al. Primary monophasic synovial sarcoma of the kidney: A case report and review of literature. Clin Med Insights Oncol. 2013;7:25762. doi: 10.4137/CMO.S12243. [PubMed: 24137053]. [PubMed Central: PMC3795529].

8. Schoolmeester JK, Cheville JC, Folpe AL. Synovial sarcoma of the kidney: A clinicopathologic, immunohistochemical, and molecular genetic study of 16 cases. Am J Surg Pathol. 2014;38(1):60-5. doi: 10.1097/PAS.0b013e31829b2dod. [PubMed: 24061517].

9. Iacovelli R, Altavilla A, Ciardi A, Urbano F, Manai C, Gentile V, et al. Clinical and pathological features of primary renal synovial sarcoma: Analysis of 64 cases from 11 years of medical literature. BJU Int. 2012;110(10):1449-54. doi:10.1111/j.1464-410X.2012.11105.x. [PubMed: 22452647].

10. Paudyal B, Paudyal P, Tsushima Y, Oriuchi N, Amanuma M, Miyazaki $\mathrm{M}$, et al. The role of the ADC value in the characterisation of renal carcinoma by diffusion-weighted MRI. Br J Radiol. 2010;83(988):33643. doi: 10.1259/bjr/74949757. [PubMed: 19620174]. [PubMed Central: PMC3473458].

11. Lv XF, Qiu YW, Han LJ, Cao J, Zhang C, Liu ZY, et al. Primary renal synovial sarcoma: Computed tomography imaging findings. Acta Radiol. 2015;56(4):493-9. doi: 10.1177/0284185114528836. [PubMed: 24682407].

12. Zakhary MM, Elsayes KM, Platt JF, Francis IR. Magnetic resonance imaging features of renal synovial sarcoma: A case report. Can- 
cer Imaging. 2008;8:45-7. doi: 10.1102/1470-7330.2008.0005. [PubMed: 18390387]. [PubMed Central: PMC2324367].

13. Young JR, Margolis D, Sauk S, Pantuck AJ, Sayre J, Raman SS. Clear cell renal cell carcinoma: Discrimination from other renal cell carcinoma subtypes and oncocytoma at multiphasic multidetector CT. Radiology. 2013;267(2):444-53. doi: 10.1148/radiol.13112617. [PubMed: 23382290].
14. Choi YA, Kim CK, Park SY, Cho SW, Park BK. Subtype differentiation of renal cell carcinoma using diffusion-weighted and blood oxygenation level-dependent MRI. AJR Am J Roentgenol. 2014;203(1):W78-84. doi: 10.2214/AJR.13.11551. [PubMed: 24951231].

15. Thway K, Fisher C. Synovial sarcoma: Defining features and diagnostic evolution. Ann Diagn Pathol. 2014;18(6):369-80. doi: 10.1016/j.anndiagpath.2014.09.002. [PubMed: 25438927]. 